\section{LA PARTICIPACIÓN PARTIDARIA Y \\ EL IMPACTO DE LOS CONTEXTOS POLIITICOS. UN ESTUDIO DE BIOGRAFÍAS MILITANTES EN JUJUY}

\author{
THE PARTY PARTICIPATION AND THE IMPACT OF \\ POLITICAL CONTEXTS. A STUDY OF MILITANT \\ BIOGRAPHIES IN JUJUY
}

\section{ADRIÁN BERARDI ·}

Docente en las Universidades Nacionales de Buenos Aires y de General Sarmiento e Investigador del Instituto de Altos Estudios Sociales de la Universidad Nacional de San Martín, Argentina.

E-mail: adrianberardi@gmail.com

\section{Resumen}

En este artículo proponemos dar cuenta de la forma en que los clivajes políticos influyen en las biografías militantes; nos preocupa responder si el contexto político constituye un elemento a tomar en cuenta para el mantenimiento del compromiso militante y de qué forma. Para llevar adelante estos objetivos analizaremos las trayectorias de dos militantes partidarios que comenzaron su participación política en la década del 2000, retomando la perspectiva de carrera propuesta por Howard Becker, considerando la trayectoria como un modelo de secuencias ordenadas de comportamiento social, tomando en cuenta los aspectos objetivos y subjetivos (la forma en que relata su propia historia).

\section{Registro bibliográfico}

BERARDI, ADRIÁN «La participación partidaria y el impacto de los contextos políticos. Un estudio de biografías militantes en Jujuy", en: ESTUDIOS SOCIALES, revista universitaria semestral, año XXVIII, n 55 , Santa Fe, Argentina, Universidad Nacional del Litoral, julio-diciembre, 2018, pp. 11-26.

\section{Abstract}

In this article we propose observe the way in which the political cleavages influence the militant biographies; we are concerned to answer if the political context is an element to be considered for the maintenance of the militant commitment and in what form. To carry out these objectives we will analyse the trajectories of twoparty militants who began their political participation in the decade of the 2000 , recovering the career perspective proposed by Howard Becker, considering the trajectory as a model of orderly sequences of social behaviour, considering the objective and subjective aspects (the way they narrate their own history).

\section{Descriptores · Describers}

clivaje político / Jujuy / militancia / trayectorias Political cleavage / Jujuy / militancy / trajectories

Recibido: 07 / 07 / 2017 Aprobado: 24 / 01 / 2018 


\section{INTRODUCCIÓN}

En las páginas que siguen daremos cuenta de la forma en que el contexto y los clivajes políticos impactan en las trayectorias de los militantes partidarios en la provincia de Jujuy. Nuestro objetivo es indagar de qué manera las decisiones que tomaron los sujetos a lo largo de su militancia fueron influenciadas por el contexto sociopolítico en el cual desarrollan su actividad; pero al hacerlo pretendemos vincular la perspectiva objetiva de los hechos históricos y la mirada subjetiva del militante. De esta forma, y más allá de mostrar de los factores motivacionales de la militancia y la configuración de redes interpersonales que permiten su sostenimiento, nos preocupa responder si el contexto político constituye un elemento a tomar en cuenta para el mantenimiento del compromiso militante y de qué forma.

Para dar cuenta de ello, analizaremos las trayectorias de dos militantes que comenzaron su participación política en la década del 2000, con el objetivo de auscultar la forma en que la política jujeña influyó en las decisiones que tomaron a lo largo de su biografía militante. Por un lado, reconstruiremos la historia militante de Marianela, que en el año 2008 comenzó a militar en la Juventud Peronista (JP) del Partido Justicialista (PJ) de Jujuy. Fue colaboradora y asesora en la legislatura provincial de varios referentes locales del partido como Eva Cruz, Liliana Fellner, Guillermo Jenefes y Germán Fellner. Desde el año 2012 integra el Consejo Provincial de la Juventud del PJ, donde alcanzó el cargo de coordinadora en 20I5. Por el otro, recuperaremos la historia de militancia de Martín en la Juventud Radical (JR) de la Unión Cívica Radical (UCR) iniciada en 2006 a través de su paso como coordinador del Programa Integral de Adolescentes de la Municipalidad de San Salvador y luego, desde fines de 2015 y a partir del triunfo electoral de Gerardo Morales, como director de Transporte Educativo, responsable del programa Boleto Estudiantil Gratuito Universal y Provincial, dependiente de la Secretaría de Equidad Educativa del Ministerio de Educación provincial y presidente de la Juventud Radical de Jujuy, distrito capital.

En este trabajo retomaremos la perspectiva de carrera de BECKER (2OI2), considerando la trayectoria como un modelo de secuencias ordenadas del comportamiento social, prestando atención tanto a los aspectos subjetivos (la mirada del militante) como a los aspectos objetivos. Considerar la perspectiva de carrera como una herramienta teórica metodológica es un punto fundamental para el estudio de la militancia, principalmente porque un análisis de tipo secuencial permite comprender que la militancia es un proceso social dinámico (FILlieule, 200I), 
que se encuentra atravesado por un conjunto de factores que puede ir modificando no sólo las prácticas y el compromiso del militante a lo largo del tiempo sino también, y sobre todo, su perspectiva respecto a su propia actividad militante.

Metodológicamente se recurre a entrevistas en profundidad a partir de la técnica del relato de vida, en tanto esta estrategia permite exponer la dimensión temporal de la trayectoria del militante y mostrar distintos acontecimientos por los que atravesó, pero desde una perspectiva en la que es el propio sujeto quien se convierte en el narrador de la historia de la sociedad de la que forma parte (SALTALAMACCHia, I987).

En este sentido:

«el relato de vida es un instrumento clave para dar cuenta de la red continua de interpretaciones subjetivas que guía la conducta de las personas, siempre que las razones para actuar sean analizadas, primero, ajustando cada paso de la carrera entre una decisión subjetiva y las coacciones objetivas. [Y] en segundo lugar [considerando] que los motivos formulados en el momento de la entrevista son producto de las reglas del juego dentro del contexto en que se expresan» (FILLIEULE, 200I: 205, traducción propia).

Cada una de las entrevistas fue seleccionada a partir del momento de iniciación de su militancia (en la década de los 2000), su estatus y condición de referente del espacio en el que participaba al momento de la entrevista. A estos fines se consideró la apreciación de distintos informantes claves. Conceptualmente, el estatus militante debe ser entendido como una categoría emergente que se vincula a la estima pública y el reconocimiento de los militantes tanto fuera como dentro de la organización a la que se integra. Es posible dar cuenta de este carácter por medio de: I) la exposición pública en los medios de comunicación, 2) el reconocimiento de otros militantes en charlas informales o entrevistas previas 3) triunfos en contiendas electorales y 4) lugar ocupado dentro de la estructura organizativa del espacio en que participa. Cada una de estas trayectorias será analizada por medio de tres dimensiones: I) motivaciones para el ingreso en la participación militante; 2) influencias y redes sociales; 3) rol político y actividades militantes. Este trabajo forma parte de los resultados preliminares de una investigación más amplia conducida en la provincia de Jujuy, por lo cual hemos incorporado referencias a otras entrevistas y charlas informales en el desarrollo del trabajo de campo. 


\section{LA PERSPECTIVA DE CARRERA: UNA FORMA DE ENTENDER LA MILITANCIA}

Desde la transición democrática la argentina, la militancia fue foco de estudio de las Ciencias Sociales y centró sus indagaciones a partir de dos factores: el estudio de los espacios de participación y de los ciclos de protesta. Estas indagaciones tendieron a vincular las condiciones generales de la militancia por medio del análisis de la estructura en la cual se desarrolla la práctica política, considerando los contextos políticos y económicos a partir de una mirada de la política nacional hacia todo el país y desatendiendo las particularidades de los diferentes contextos regionales.

La perspectiva de carrera (BECKER, 2OI2) propone una entrada al estudio de la militancia a partir del propio militante, recuperando su punto de vista respecto de su participación militante al tiempo que establece una herramienta teórica metodológica para el estudio de la trayectoria de las personas dentro de un ámbito específico a partir de un análisis secuencial de las acciones que llevan adelante los individuos a lo largo de su biografía. Cada una de estas instancias está determinada por un proceso de contingencia, a partir del cual el sujeto aumenta o disminuye su compromiso. Por otra parte, el estudio de la carrera abarca el desarrollo de un proceso de aprendizaje dentro de las actividades que se desarrollan, e involucra no solo un conjunto de prácticas y regularidades propias del ámbito militante, sino también la conformación de relaciones entre los viejos y los nuevos militantes. De esta forma, se constituye como una herramienta para el estudio de la trayectoria militante con un carácter dinámico, en la medida en que el recorrido puede cambiar y generar nuevas identidades y significados a lo largo del tiempo (BECKer y STRAuss, 1956), dando cuenta de la condición de movilidad durante la historia militante.

La conducción de este tipo de estudio abarca tanto una dimensión objetiva, que permite dar cuenta de las secuencias típicas a partir de posicionamientos de estatus o jerarquías, como una dimensión subjetiva, necesaria para interpretar el significado que las personas les otorgan a las acciones que llevaron adelante en cada una de las etapas a lo largo de su biografía (hughes, 1958). Por último, partiendo de la perspectiva de FILlieule (20OI), entendemos a la militancia como un proceso social dinámico, individual y colectivo, que implica el establecimiento de un compromiso a una causa. Este proceso de involucramiento interpela al sujeto generando la puesta en marcha de un conjunto de acciones, por medio de distintas estrategias, que se llevan adelante y que implica una conexión entre los aspectos objetivos y las condiciones subjetivas. Por otra parte, la propuesta de análisis de 
trayectorias militantes de Jujuy nos permitirá dar cuenta de la importancia del contexto subnacional y la influencia o no del contexto nacional.

\section{LAS TRAYECTORIAS DE MILITANTES PARTIDARIOS DE JUJUY}

Marianela nació en San Salvador de Jujuy en 1990 y desde chica tuvo una inquietud particular por la participación política. En la escuela secundaria decidió involucrarse en la organización de la fiesta del estudiante, fue delegada de su curso y formó parte de la comisión estudiantil. Cuando terminó el colegio secundario y comenzó a estudiar el profesorado de Ciencias Políticas en el Instituto Populorum Progressio, decidió iniciar su militancia política y en el año 2008 ingresó a la Juventud Peronista (JP) del Partido Justicialista (PJ) de Jujuy; quizás influenciada por su abuela peronista (aunque sus padres no querían saber nada con la idea de que milite). Fue en ese momento que junto con otros compañeros (también de la JP) comenzaron a organizar el centro de estudiantes, que llegó a presidir.

Martín es jujeño y su madre, una militante de la UCR lo dio a luz en I988; también fue ella quien a los I8 años lo invitó a participar en el partido. En el año 2006, Martín ingresó a la JR de la UCR, pero sus intereses no estaban en la militancia política sino en la abogacía, por eso relegó su participación partidaria y se fue a estudiar Derecho a la Universidad Nacional de Tucumán; sin embargo, los resultados iniciales de su carrera universitaria no alcanzaron sus expectativas y volvió a Jujuy. En San Salvador comenzó a estudiar Comunicación Social y se involucró en Franja Morada (organización juvenil universitaria de la UCR), pero volvió a abandonar sus estudios universitarios al poco tiempo, y retomó su militancia en la JR.

A pesar de que ambos son militantes de partidos tradicionales de la Argentina y comparten una misma generación, la forma y las motivaciones para involucrarse en la militancia fueron diferentes; así recuerda Marianela ese momento:

«Me acuerdo clarito un diciembre con otra compañera salíamos de tener clases y estaba tomada la plaza y estaba Milagro [Sala] haciendo la Pachamama en la puerta de la Casa de Gobierno, y con ella dijimos ‘bueno, vamos a hablar, vamos a ver qué dice; llegamos a Milagro y le preguntamos ‘qqué es lo que pasa?), y me acuerdo que ella nos miró y nos dijo (¿̨ustedes de dónde son?), «no, estudiamos Ciencias Políticas, somos del Populorum; ‘bueno, vengan a ver lo que voy a hacer, y fue y tomó la 
Legislatura, o sea, fue magnífica la experiencia, porque ella entró, ella sola abrió la puerta, estaban unos policías ahí, no le dijeron nada obviamente, ella pasó con un grupo de gente llevando colchones, tiraron los colchones en el medio de la sala de la Legislatura y empezó la toma. La verdad que después que salimos de eso, ella nos trató muy bien, pudimos hablar, ella nos explicó cuál era el motivo de lucha, nos dijo que no encontraba otra forma de diálogo con el entonces gobierno del doctor [Walter] Barrionuevo, y por ahí nosotros empezamos a entender que para poder estar dentro y para poder empezar a cambiar las cosas teníamos que sí o sí estar en los lugares de toma de decisiones. (...) Milagro fue un hecho, digamos, un fenómeno social y político para mí que marcó mucho mi forma de ver la política jujeña» ${ }^{1}$.

Milagro Sala se constituyó en un punto de referencia de Marianela, pero también fue un actor central de la política jujeña: siendo delegada sindical de la Asociación de Trabajadores del Estado (ATE), Sala creó en 1998 la Organización Barrial Tupac Amaru como una pata barrial de la Central de Trabajadores Argentinos de Jujuy, a partir de la implementación de la copa de leche. Desde el ańo 2003, con la puesta en marcha del Plan de Emergencia Habitacional por iniciativa del gobierno de Néstor Kirchner, la organización creó cooperativas de trabajo para la construcción de viviendas; así desarrolló una gran capacidad organizativa y de administración de recursos; al mismo tiempo que produjo una fuerte interacción entre movilización (exigiendo recursos al Estado provincial para los sectores desocupados), inserción en los barrios y el liderazgo de Sala (BATTEZZATI, 2OI4 y мOsCOVICH, 2009). La Tupac Amaru no solo tuvo la capacidad de generar empleo y garantizar derechos sociales para los sectores más vulnerables de la provincia, sino que además se configuró como opositora al gobierno provincial y desplazó a otras organizaciones sociales de la representación de los pobres y marginados de la provincia de Jujuy.

Por otra parte, Marianela hace notar en su relato que el conflicto en el que vio actuar a Milagro se dio durante la gobernación de Walter Barrionuevo (2007-20II) y no es un dato menor. Eduardo Fellner había sido gobernador de transición en 1998, luego de la renuncia de su antecesor Carlos Ferraro, en medio de una fuerte crisis social producto del desempleo y la pobreza en la provincia donde al mismo tiempo se vivía un ciclo de importante inestabilidad política e ingobernabilidad

\footnotetext{
${ }^{1}$ Entrevista a Marianela, PJ, marzo de 2016.
} 
(LAGOS Y GUTIÉRREZ, 2006; LAGOS y CONTI, 20IO). Fellner fue reconocido por los miembros del pu provincial como el único capaz de lograr la unidad al interior del partido a partir de su lugar como gobernador, esto le valió ser electo para el Ejecutivo provincial en 1999, 2003 y 2011.

En este sentido, Carla, militante del PJ provincial, exdiputada nacional y expresidenta de la JP, afirma:

«si algo hay que reconocerle a Fellner en aquellos años de anarquía total es que él con un juego de negociación, búsqueda de consenso y diálogo permanente con toda la dirigencia peronista, fue cerrando acuerdos (...) él ordenó la dirigencia, logró contener a todos y consolidar un liderazgo» ${ }^{2}$.

Sin embargo, imposibilitado de una tercera reelección y sin respaldo para una reforma constitucional en la provincia, Fellner decidió elegir como su sucesor a Barrionuevo a pesar de la oposición de otros sectores del partido, rompiendo el diálogo intrapartidario; para muchos este acontecimiento fue fundamental para entender los últimos ańos en la provincia. Barrionuevo representó la imposibilidad de la continuidad del diálogo al interior del partido y generó un nuevo fraccionamiento dentro del pj de Jujuy, dando origen al Frente Primero Jujuy (integrado por fracciones del PJ, sectores de la UCR y partidos provinciales); pero también fue acusado de haber abandonado las bases sociales del partido y haber provocado un aumento sustancial de la protesta social, sobre todo a partir de no permitir la interacción con organizaciones sociales como la Tupac Amaru. En el caso de Martín, la influencia del contexto en su involucramiento fue secundaria:

«Yo mamé la identidad radical, vos imaginate que yo debo haber tenido 7 años y mi madre era militante, entonces recuerdo haber salido por Alto Comedero, que es uno de los barrios más grandes de acá de San Salvador de Jujuy, a repartir votos por debajo de la puerta, que se estilaba o se estila todavía hacer el voto bajo la puerta, [pero] yo me acerqué cuando dejé de estudiar para decir ‘bueno andá a hacer algo, andá a fijarte si podés conseguir un trabajo, andá a militar por lo menos` (...) a mí lo que me acercó fue justamente la necesidad económica, pero después me hizo apasionar, y uno hizo de esto un estilo de vida ${ }^{3}$.

\footnotetext{
2 Entrevista a Carla, PJ, marzo de 2016.

${ }^{3}$ Entrevista a Martín, UCR, septiembre de 2016.
} 
Luis, militante de la Juventud Radical, sostiene que en muchos casos el acercamiento a la militancia pasa por otro lado que no siempre se vincula, inicialmente, al interés por la política: «Yo empecé a militar a los I6 ańos participando en las pegatinas para las campañas, nos convidaban gaseosa, nos daban algo para comer, unos sándwiches (...) después me empezó a gustar, te intriga, te llama todo esto de la militancia, y empezás» ${ }^{4}$.

Retomando las trayectorias de Martín y Marianela, podemos observar, a pesar de las diferencias en la importancia del contexto político en el momento de involucrarse a la militancia partidaria, que en ambos casos hay un fuerte proceso de intercontingencia en el cual los dos militantes son motivados por otras personas en el momento de iniciar su militancia. En este sentido, es importante comprender que el proceso de involucramiento en muchos casos está mediado por las redes sociales o interpersonales, configuradas a partir de vínculos que construyen los sujetos, y que se convierten en relaciones que facilitan e influyen en el involucramiento (DIANI, 2004; DELla PORTA y DIANI, 2OII; MCADAM, I982 y I988). Por otra parte, son esas mismas redes interpersonales las que le permite al militante ir construyendo un proceso de aprendizaje a partir del cual configurar sus prácticas dentro de la militancia, es decir que no solo existe un vínculo que genera un proceso de influencia, sino que la propia práctica construye y reconstruye nuevas redes que permiten regenerar constantemente ese compromiso (KITTS, 2000). En el caso de Marianela, el proceso de intercontingencia en su trayectoria militante no solo estuvo determinado por el contexto jujeño, sino también por el contexto sociopolítico a nivel nacional; sobre todo por las figuras de Néstor Kirchner y Cristina Fernández de Kirchner quienes, desde la mirada de Marianela, retomaron los lineamientos del «peronismo como ideología política y el justicialismo como doctrina social». Alejandro, militante del Partido Justicialista de la provincia, también reivindica el peso del kirchnerismo, principalmente enfocando en la oportunidad política que generaron sus gobiernos para la juventud militante:

«Néstor y Cristina, Néstor sobre todo, trajo ese aire, como dicen todos, del sur, y creo que dejó como legado el tema de que pueden llegar a empoderarse por las vías legales. Antes de ellos no había tantos canales de participación tan abiertos como ahora, la juventud

${ }^{4}$ Entrevista a Luis, UCR, septiembre de 2016. 
pasó a ser un actor social importante está dentro de lo que es la agenda política, y es tenida en cuenta como un factor, como algo que podía llegar a tener lugares de decisión» ${ }^{5}$.

Para Martín, por su parte, fue fundamental conocer los valores que aportó Raúl Alfonsín a la doctrina radical, aunque no vivió su gestión como presidente de la Argentina: «Leí mucho sobre Alfonsín y sin duda sus ideas aportaron a mi formación dentro del partido, pero esto pasó cuando yo ya militaba y comencé a estudiar la doctrina radical». Otros militantes radicales sostienen que el peso y la influencia de Raúl Alfonsín constituyen uno de los principales pilares de la identidad radical, principalmente para los jóvenes que se vincularon al partido después de 1983. José, militante de la UCR y funcionario del gobierno provincial, afirma que "Alfonsín era un personaje que subyugaba, transmitía convencimiento» ${ }^{6}$.

Más allá de sus inicios y motivaciones, las dos trayectorias están atrapadas en un recorrido dentro de las estructuras partidarias y los distintos espacios de gestión gubernamental que cada uno ocupó. Marianela comenzó a trabajar en el Instituto de Vivienda del gobierno provincial cuando como estudiante de Ciencia Política obtuvo una beca. Esa pasantía le permitió acceder posteriormente a un puesto en el Ministerio de Desarrollo Social bajo la gobernación de Eduardo Fellner. En tanto, partidariamente, en el año 2012 pasó a formar parte del Consejo Provincial de la Juventud del pu (bajo la conducción de Silvina Sadir), un espacio creado "por una iniciativa de Eduardo Fellner», sostiene Marianela, del que llegó a ser coordinadora en 2015.

Por su parte, Martín comenzó a trabajar en la municipalidad de San Salvador de Jujuy bajo la intendencia del radical Raúl «Chulli» Jorge, y desde allí accedió a ser coordinador del Programa Integral de Adolescentes de la Municipalidad. Con el triunfo electoral de Gerardo Morales como gobernador de la provincia en 2015, Martín fue nombrado director de Transporte Educativo y responsable del programa Boleto Estudiantil Gratuito Universal y Provincial, dependiente de la Secretaría de Equidad Educativa del Ministerio de Educación provincial. Y en el 2016 llegó a la presidencia de la Juventud Radical de Jujuy en el distrito capital.

Marianela, a diferencia de Martín, no siguió un camino laboral dentro del Poder Ejecutivo, sino que comenzó a trabajar como colaboradora y asesora de varios dirigentes del partido en la legislatura provincial, entre ellos Eva Cruz,

\footnotetext{
${ }^{5}$ Entrevista a Alejandro, PJ, marzo de 2016.

${ }^{6}$ Entrevista a José, UCR, septiembre de 2016.
} 
Liliana Fellner, Guillermo Jenefes y finalmente Germán Fellner, consolidando su lineamiento político con el fellnerismo y afirmando la estrecha relación entre este sector y la JP. Este vínculo entre el sector fellnerista del pj y la Juventud Peronista, también es relatado por Alejandro:

«Fellner empezó de alguna manera a darle más participación a la parte juvenil, le dio más bolilla, antes no se le daba bolilla, con el doctor Fellner cambió de a poco, abrió un poco los canales de participación de jóvenes, nosotros la verdad que nos sentíamos un poco más contenidos, no era alguien que era imposible de llegar a él, de charlar, de tocar la puerta para hablar con él, se sentía un poco más accesible, a diferencia de la otra época nefasta que se vivió» (Alejandro).

Retomando las biografías militantes de Marianela y Martín, notamos que nuevamente la diferencia en el desarrollo de sus actividades da cuenta de trayectorias distintas; sin embargo, hay un punto en común. Ambos, como militantes partidarios, identifican dentro de sus tareas la participación en las campañas electorales; no obstante, tienen posiciones contrapuestas respecto a estas prácticas:

«En 2013 Silvina Sadir fue candidata a diputada nacional, y empezamos con una responsabilidad mucho más importante, así que bueno, empezamos a laburar, los actos, la campaña era bastante dura, encabezaba la lista Oli Tentor, que es el actual diputado nacional. La verdad que una experiencia muy buena, muy enriquecedora, pero sí, la verdad que fueron como ańos bastante intensos» (Marianela).

«En su momento me tocó salir a hacer pegatinas, hacíamos todos, me acuerdo la campaña del 20I3, todo lo que es folletería, los pasacalles los hizo la juventud, pero le dimos una vuelta de rosca, porque decíamos, a ver, nosotros no estamos solamente para venir a pegar unos afiches o para venir a pintar un pasacalle, si no es como que somos la mano de obra barata de un partido, de un partido centenario que tiene que sacar nuevos militantes para lo que se viene, para la nueva política»» (Martín).

Como podemos observar, para Marianela participar en la campaña electoral se constituyó en un proceso de aprendizaje y un compromiso a partir del cual pudo dar cuenta de su capacidad como cuadro político del partido, y encontrar en ese tipo 
de actividad un canal de construcción política. En este mismo sentido, se expresa Carla, militante del PJ provincial, exdiputada nacional y expresidenta de la JP:

«Cada campańa yo venía con tiempo (me tomaba tres o cuatro semanas, tenía permisos especiales en la universidad) y me iba involucrando en las campańas cada vez más. Ahí empiezo yo a militar en la Juventud Peronista, y entre tanta campaña ya había armado ya un grupo de Juventud Peronista en mi localidad, entonces participé dentro de una lista provincial» (Carla).

Sin embargo, Martín entiende que la participación en la campańa electoral era una tarea que podía hacer cualquiera, y que si lo hacía la juventud era principalmente para ningunear a los jóvenes y no brindarles posibilidades dentro de los espacios de toma de decisiones:

«la Juventud no era eso nada más, no era eso, teníamos que ver cómo podíamos llegar nosotros a ocupar, estar realmente en el plano político, entonces empezamos con lo que era formación, dijimos acá la mano de obra barata se acabó, si hay que hacerla la vamos a hacer, nos hicimos de enemigos internos a propósito con el fin de estar en un plano político, con el fin de que nos nombren, con el fin de que digan «mirá aquellos pendejos de mierda qué atrevidos que son, ya quieren dar el paso y en 2009 conseguimos meter dos funcionarios en la Municipalidad» (Martín).

La lectura de Martín respecto al rol de la juventud en el partido y su lugar en las elecciones también es retomada por Luis:

«En el tiempo de elecciones, eran dos o tres meses que me quedaba, me bañaba y dormía en el comité prácticamente, pero no siempre era reconocido mi trabajo. Por eso cada vez que se suma un joven y me dice «che, mirá, me quiero sumar a la Juventud, 〈sí, de diez〉, le digo, y yo cuando los veo que están muy entusiasmados y quieren dar todo, digo ‘tené cuidado porque en algún punto todo el sacrificio por ahí no es muy valorado, así que hacelo por parte, andá despacio»»"

${ }^{7}$ Entrevista a Luis, UCR, septiembre de 2016. 
En este sentido, y a partir de su planteo, Martín da cuenta de sus intereses para poder avanzar en la construcción política hacia el interior del partido, para de esa forma poder acceder a espacios de representación y de toma de decisiones; por eso él considera que no era posible disminuir la militancia de la juventud solo a campańas, sino que por el contrario había que lograr que los jóvenes tengan capacidad de representación dentro del partido y en la provincia. Así, es interesante entender la forma en que cada uno interpreta la construcción política, las secuencia. La idea de construcción política, desde estas perspectivas, hace referencia a la conformación de vínculos, partidarios y extras partidarios, que faciliten al militante construir una imagen pública capaz de ubicarlo en la consideración para la ocupación de cargos en espacios de toma de decisiones o la participación de procesos electorales.

Por otra parte, a pesar de que para Martín la participación en la campaña electoral, sin formar parte de listas que compitan por un cargo, constituye un ninguneo a los sectores juveniles, distintos entrevistados coinciden en sostener que en las trayectorias militantes partidarias este tipo de prácticas no solo constituye un proceso de conocimiento del mundo de la política, sino también un proceso de aprendizaje. Para Marianela, esas prácticas deben asumirse como pasos necesarios para la construcción política, que se deben asociar a los lugares que la juventud ocupa dentro del partido, entendiendo que son condiciones de la propia construcción y que en todo caso es la capacidad de esa construcción la que genera nuevos espacios en la toma de decisión decisiones para ser ocupados por los miembros del partido que en principio están excluidos:

"como coordinadora provincial de la Juventud estoy en un lugar en el que de verdad tenés la posibilidad de tomar decisiones y donde tenés la oportunidad de elaborar realmente políticas públicas para los jóvenes (...) y estoy segura que en algún momento vamos a poder estar en los momentos claves de decisiones políticas, que cambien el destino de la patria; pero para eso primero es importante que renueven las autoridades partidarias y de ahí en más poder hablar de unas elecciones internas de la rama de Juventud Peronista; pero dentro de un proyecto político» (Marianela).

Volviendo al impacto de los clivajes políticos en las trayectorias, es posible dar cuenta que, a pesar de que ambos ocupan lugares de importancia en la estructura partidaria, Martín como presidente de la Juventud Radical de Jujuy distrito capital y Marianela como coordinadora de la Juventud del pJ, sus biografías son 
alteradas constantemente por el proceso histórico que atraviesan en el momento en que fueron entrevistados.

Marianela forma parte de la oposición al gobierno del radical Gerardo Morales, y Martín no solo es militante del partido gobernante, sino que también es funcionario público de dicha gestión. Así, la performance electoral de sus respectivos partidos les facilitó el acceso a distintos espacios de toma de decisiones y/o de gestión, donde además de poder acceder a recursos económicos, les permitió llevar adelante sus respectivas construcciones políticas. Por otra parte, en los dos casos la idea de construcción política tanto interna como externa al partido está latente como una característica fundamental de su militancia, y sin duda la diferencia se encuentra en el contexto en que se inscribe su relato. Sin embargo, en la reconstrucción biográfica de Martín surge un dato interesante; en el momento de relatar el impacto del contexto jujeño en su historia militante, él sostiene: «capaz que, si perdíamos las elecciones en el 2007, yo hoy día no seguía haciendo política, capaz que estaba buscando laburo en un comercio, capaz que volvía a arrancar mi carrera de estudiante». Esta afirmación nos permite indicar dos puntos fundamentales en ambas trayectorias, el primero la forma en que el contexto político se configuró como una oportunidad política para Martín y terminó consolidando su compromiso militante, a pesar de no ser necesariamente el mismo contexto el motivador de su involucramiento.

Por otra parte, la trayectoria de Marianela atravesó un punto de fractura, o contingencia (BECKER, 20I2), justamente a partir de la derrota electoral del PJ en 2015 y la pérdida del pu de la gobernación de la provincia después de 32 años. En cierto punto, la perspectiva de cada uno de los militantes también se encuentra inscripta en la estructura política en la cual desarrollan sus prácticas, principalmente si se entiende que el Partido Justicialista de Jujuy desde 1983 tuvo el control político de la provincia, en tanto la UCR desde la transición democrática siempre ocupó el lugar de segunda fuerza política en el entramado institucional.

En este sentido, Marianela sostiene:

«la verdad que es bastante complicado ser oposición, en Jujuy particularmente no estamos acostumbrados, no podemos terminar de saber cómo ser oposición (...) pero a pesar de eso las experiencias que uno va teniendo son muy grandes, pero por ahí lo más importante para mí es resaltar que uno puede militar con convicción y con ideales, creo que eso es lo más importante, inclusive sin tener laburo, inclusive 
haciendo o rebuscándola para poder conseguir algo, teniendo o no teniendo físicamente ese conductor que uno quiere o que aprecia o que espera, y podemos seguir transformando la realidad».

\section{ALGUNAS CONCLUSIONES}

La perspectiva de carrera constituye un punto importante para comprender la forma en que los militantes analizan sus propias trayectorias, lo fundamental es la manera en que justifican sus decisiones dentro del conjunto general de sus prácticas. En este sentido, se procuró aquí dar cuenta de la forma en que los clivajes políticos significaron, en la trayectoria de los militantes que aquí presentamos, un elemento de importancia para la continuidad de su compromiso político. Los factores de involucramiento y los intereses que motivaron su ingreso a la militancia también se encuentran muy presentes en los relatos, y muestran cómo el contexto político, tarde o temprano, juega un rol fundamental en la trayectoria militante.

Sin embargo, el eje nodal de nuestro análisis y la descripción de las trayectorias nos permiten sostener que el ámbito provincial, y los clivajes políticos a partir de los procesos electorales, constituyen el elemento principal de las oportunidades políticas.

Si bien es posible entender la existencia de componentes motivacionales externos al contexto, el involucramiento militante requiere de otro punto fundamental, la oferta de participación (KLANDERMANS, 2004); es decir los espacios militantes que los sujetos consideren necesarios para poder trabajar sobre sus intereses y sus inquietudes. Pero, al mismo tiempo, esa oferta de participación necesita poder brindar resultados positivos a las búsquedas que orientan esos intereses.

Así, podemos ver claramente cómo en el caso de Martín el partido radical jugó un rol central al momento de encontrar una alternativa a sus vicisitudes estudiantiles; en tanto, en el caso de Marianela podemos dar cuenta cómo el PJ funcionó como una válvula de escape a sus intereses políticos que se fueron generando en su adolescencia. En ambos casos, sin duda, la búsqueda de ocupar espacios de decisión con el objetivo de cambiar la realidad constituye su principal objetivo; sin embargo, justamente la meta de esa militancia debe ser entendida como el punto de contingencia de la trayectoria militante.

Poder alcanzar el objetivo no solo depende del militante, o de la oferta de participación, sino principalmente del contexto político en el cual el espacio de 
involucramiento militante se encuentra inscripto. El momento en que el pJ logró ocupar el gobierno provincial, o sus referentes llegar a cargos legislativos, le permitió a Marianela configurar su propia construcción política; lo mismo sucedió en la biografía de Martín, con la diferencia que para él una derrota electoral podría haber sido el fin de su militancia. Al mismo tiempo, si acceder al involucramiento militante requiere de un conjunto de redes o vínculos sociales que relacione al militante con las estructuras de participación, el contexto sociopolítico hace de ese espacio de participación un lugar posible o no. Las probabilidades de alcanzar los objetivos que abre el partido político, es decir las oportunidades políticas que surgen dentro del partido, se encuentran determinadas fundamentalmente por la capacidad electoral que garantiza el ingreso a espacios de toma de decisión.

De esta forma, mientras que el propio contexto de movilización jujeño fue un factor motivacional del involucramiento de Marianela en la militancia política (aunque no fue así para Martín), los resultados electorales fueron los que realmente generaron nuevos espacios de participación para ambos militantes. Aunque la mirada sobre estos procesos esté más clara en una trayectoria (la de Martín) que, en la otra, queda claro que cuando el partido logró acceder al manejo de la gestión ejecutiva -sea una gobernación o un intendencia- los militantes pudieron ocupar espacios en la toma de decisiones y avanzar dentro de la estructura partidaria. El contexto político no solo debe ser interpretado como un factor motivacional en el momento de iniciar una trayectoria militante partidaria (incluso puede no serlo); sino, y por sobre todas las cosas, como un elemento fundamental para entender la manera en que el militante puede acceder a nuevos espacios tanto en la estructura partidaria como en la política institucional, y aumentar sus niveles de compromiso. 


\section{Referencias bibliográficas}

BATTEZZATI, SANTIAGO (2014): «La Tupac Amarú: LAGOS, MARCELO Y CONTI, VIVIANA (2010): Jujuy de movilización, organización interna y alianza con la revolución de mayo a nuestros días, Jujuy, el kirchnerismo (2003-2011)», en: Población y EdiUnju.

Sociedad, vol. 21, $\mathrm{n}^{\circ} 1$, pp. 5-32.

LAGOS, MARCELO Y GUTIÉRREZ, MIRTA (2006): «DictaBECKER, HOWARD (2009): Trucos del Oficio, como conducir su investigación en ciencias sociales, Buenos Aires, Siglo XXI. dura, democracia y políticas neoliberales 19761999», en: A. Teruel y M. Lagos (Dirs.), Jujuy en la historia: de la colonia al siglo XX, Jujuy, BECKER, HOWARD (2012): Outsider, Buenos Aires, Siglo XXI.

DELLA PORTA, DONATELLA Y DIANI, MARIO (2011): LOS Movimientos Sociales. Madrid, CIS. EDIUNJU, pp. 234-294.

MCADAM, DOUG (1982): Political process and development of black insurgency, 1930-1970, Chicago, The University of Chicago Press.

DIANI, MARIO (2004): «Networks and Participation», en: D. Snow, S. Soule y H. Kriesi (edis.), The Blackwell Companion To social movements, Malden, Blackwell Publishing Ltd, pp. 339-359. KITTS, JAMES (2000): «Mobilizing in black boxes: social networks and participation in social movement organizations», en: Mobilization: An International Journal, vol. 5, $\mathrm{n}^{\circ}$ 2, pp. 241-257. KLANDERMANS, BERT (2004): «The demand and supply of participation: Social psychological correlates of participation in social movements», en: D. Snow, S. Soule, H. Kriesi (eds.), The Blackwell Companion to Social Movements, Oxford, BlackMCADAM, DOUG (1988): Freedom summer, Oxford: Oxford University Press.

MOSCOVICH, LORENA (2009): «Fronteras entre la participación y la política. Estudio de las relaciones entre los gobiernos subnacionales y las organizaciones sociales con financiamiento federal en la Argentina», en: XXI Congreso Internacional de Ciencia Política, Santiago de Chile.

SALTALAMACCHIA, HOMERO (1987): «Historia de vida y movimientos sociales: el problema de la representatividad (Apuntes para la reflexión)», en: Revista Mexicana de Sociología, vol. 49, $\mathrm{n}^{\circ}$ 1, pp. 255-277. well Publishing, pp. 360-379. 